\title{
Anatomical Variants of Coronary Venous System on Cardiac Computed Tomography
}

\author{
Rafal Mlynarski, MD, PhD; Agnieszka Mlynarska; Maciej Sosnowski, MD, PhD
}

\begin{abstract}
Background: In a few studies, huge anatomical variability of coronary venous system (CVS) has been documented without analysis of its variants. The aim of the present study was to evaluate anatomical variants of CVS in multislice computed tomography (MSCT).

Methods and Results: In 199 patients (114 males, age 56.6 \pm 11.5 years), a 64-slice computed tomography (Aquilion 64) was performed due to coronary artery disease suspicion. A scan with electrocardiogram-gating was performed using a slice thickness of $0.5 \mathrm{~mm}$ during a breath-hold. In each case, 3D volume rendering and 2D multi-planar reformatting reconstructions of CVS were created (Vitrea 2). As target veins for cardiac resynchronization therapy (CRT), the posterolateral, lateral and anterolateral veins were recognized. Coronary sinus was well visualized in all cases. A total of 27 anatomical variants of CVS were identified, 9 of them are most common (in $148 / 199$ cases; $74.4 \%$ ). In 4 out of these 9 variants, a single coronary vein in the target area for CRT appeared, 2 target veins in 3 variants and 3 veins in 2 variants occurred. In 6 cases ( 2 seldom variants $-3.0 \%$ ) no veins in the target area were identified.
\end{abstract}

Conclusions: In the majority of the examined patients, at least one vein in the target area for CRT was observed. Anatomical variability of CVS strengthen the potential role of MSCT in CVS visualization before CRT implantation. (Circ J 2011; 75: 613-618)

Key Words: Cardiac resynchronization therapy; Computed tomography; Coronary veins

$\mathbf{T}$ he variety of cardiac devices for transvenous implantation has increased in the years, mostly due to evidence of effectiveness of this therapy in chronic heart failure. Special attention is placed on cardiac resynchronization therapy (CRT) or combination of those devices with implanted cardioverter defibrillator. ${ }^{1-5}$ During these procedures, the implantation of left ventricle lead is necessary. The only way for transvenous left ventricle lead implantation is coronary sinus (CS) cannulation from the right atrium, and finally implantation of the lead into one of the coronary veins. ${ }^{6}$ Effectiveness and success of implantation have been shown to depend mainly on a proper placement of the left ventricle electrode into the coronary target vein. ${ }^{7,8}$ Lateral, posterolateral and anterolateral veins (PLV and ALV) provide optimal hemodynamic benefits. Meanwhile, most operators are faced with the problem of huge anatomical variability of coronary veins. Knowledge of the coronary venous system (CVS) anatomy is an important factor before many electrophysiological procedures, such as CRT or ablations. The CVS was the subject of a few research groups, but most of the research came from post mortem studies. ${ }^{9,10}$ In a few studies, a huge anatomical variability of CVS was docu- mented; however, its variants have only rarely been classified. ${ }^{11}$ Recent data indicated a potential of the latest generation multislice computed tomography (MSCT) scanners for CVS anatomy visualization; ${ }^{12,13}$ however, anatomical variants with emphasis on target veins for CRT have not been examined yet.

The purpose of the present study was to evaluate anatomical variants of CVS in MSCT in the context of target veins for CRT.

\section{Methods}

A total of 199 patients (114 males, 57.3\%), aged 56.6 $6 \pm 11.5$ with suspected coronary artery disease (CAD) were included in the study. A suspicion of CAD was based on clinical symptoms such as chest pain, shortness of breath when exercising or during other vigorous activities and other typical/ atypical symptoms co-existing. Non-specific electrocardiogram (ECG) changes and positive results of exercise testing was also a basis for performing MSCT. Arterial hypertension and hyperlipidemia were the most prevalent CAD risk factors, present in $143(71.8 \%)$ and $94(47.2 \%)$ patients, respec-

Received July 27, 2010; revised manuscript received October 4, 2010; accepted November 4, 2010; released online January 14, 2011 Time for primary review: 43 days

Department of Electrocardiology (R.M., A.M.), Unit of Noninvasive Cardiovascular Diagnostics (R.M., M.S.), Upper-Silesian Medical Center, Katowice; and 3rd Division of Cardiology, Medical University of Silesia, Katowice (M.S.), Poland

Mailing address: Rafal Mlynarski, MD, PhD, Upper-Silesian Medical Center, Electrocardiology Department, Katowice 40-635, ul Ziolowa 45/47, Poland. E-mail: Rafal_Mlynarski@mp.pl

ISSN-1346-9843 doi:10.1253/circj.CJ-10-0736

All rights are reserved to the Japanese Circulation Society. For permissions, please e-mail: cj@j-circ.or.jp 


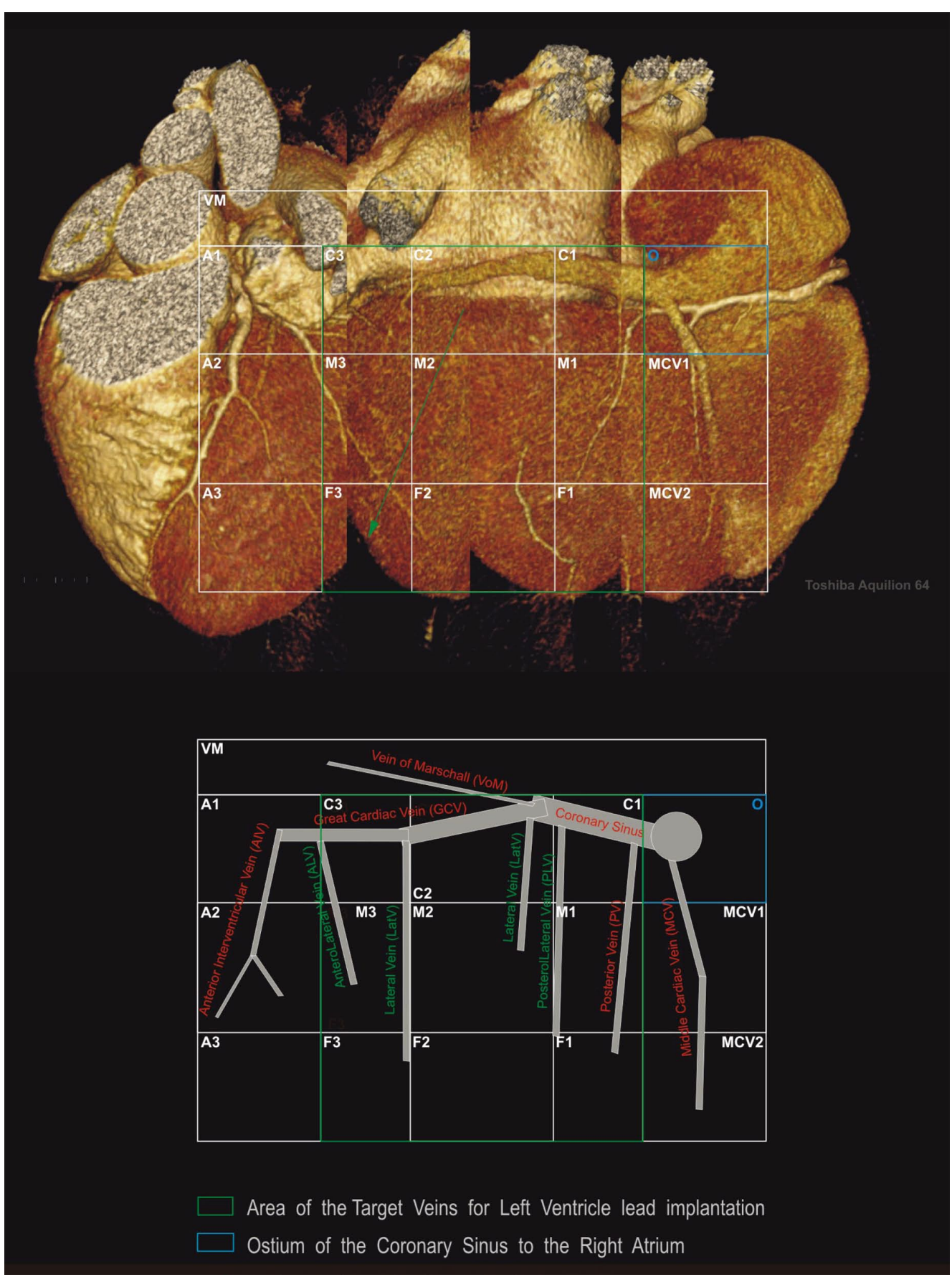

Figure 1. Multislice computed tomography analyzing matrix (own project) created to facilitate nomenclature of coronary veins.

tively. Diabetes $(57 / 114,28.6 \%)$, smoking $(75 / 114,37.7 \%)$ and cardiovascular diseases in the family $(44 / 114,22.1 \%)$ were additional risk factors. Exclusion criteria were: the presence of atrial fibrillation, frequent premature heartbeats, renal insufficiency (serum creatinine $>1.2 \mathrm{mg} / \mathrm{dl}$ ), hyperthyreosis, known allergy to non-ionic contrast agents and a previously implanted pacemaker with unipolar leads. Patients after coronary artery bypass surgery were also excluded. The local Ethics Committee approved the study protocol. Informed consent was obtained in each case.
In each patient, a 64-slice computed tomography (CT) was performed using an Aquilion 64 scanner (Toshiba Medical Systems, Tochigi, Japan). Scanning with retrospective ECGgating was performed using 64 slices with a collimated slice thickness of $0.5 \mathrm{~mm}$ during a breath-hold. A breath-hold examination was performed to adjust the scanner settings. The helical pitch was 12.8 (best mode) and rotation time was $0.4 \mathrm{~s}$. The tube voltage was strictly dependent on a patient's body mass index (BMI): for $\mathrm{BMI}<23.9$ it was $120 \mathrm{kV}$ at $330 \mathrm{~mA}$, for $\mathrm{BMI}=24.0-29.9$ it was $135 \mathrm{kV}$ at $380 \mathrm{~mA}$ and 
for $\mathrm{BMI}>30.0$ it was $135 \mathrm{kV}$ at $430 \mathrm{~mA}$. ECG-dependent dose modulation-an algorithm, which modulates the tube current according to the ECG during the scan, was not used it this study. This algorithm reduces the tube current during systole. In systolic phases quality of coronary arteries reconstructions is usually not acceptable, but quality of coronary veins reconstructions is usually optimal. ${ }^{13,14}$ A pre-selected region of interest in the descending aorta was used. The start of triggering was at 180 Hounsfield units. On average, $100 \mathrm{ml}$ of non-ionic contrast agent (Ioperamid, Ultravist 370, Schering, Germany) was given to each patient during the examination at an average rate of $5 \mathrm{ml} / \mathrm{s}$. The contrast agent was given in 3 phases: $90 \mathrm{ml}$ of contrast agent (average), then $24 \mathrm{ml}$ of contrast agent and $16 \mathrm{ml}$ of saline flush $(60 \% / 40 \%)$ and finally $30 \mathrm{ml}$ of saline. The cut-off for heart rate (HR) was set at 65 beats/min. If the HR was higher, metoprolol succinate (Betaloc, Astra Zeneca, Sweden) at a dosage of 5-10 mg was administered intravenously, if not contraindicated. If the expected HR slowing was not achieved, the patient was excluded from the study. Sublingual nitroglycerin was not given before image acquisition.

Reconstructions of data were performed on Vitrea 2 (software version 3.9.0.0) workstations (Vital Images, USA). In each case in the study presented, 10 axial image series, as well as $3 \mathrm{D}$ volume rendering reconstructions, using a $2.0 \mathrm{~mm}$ slice thickness to reduce a large amount of data, were created from $0 \%$ to $90 \%$ R-R intervals (step 10\%). Our earlier experience from results for a large group of patients, showed that in most cases the quality of the visualization of coronary veins is the best at the systolic phase (30-40\% R-R interval), and in some patients at the $50 \%$ phase. In a small group of patients $(10.1 \%)$, the best results were obtained at other phases. To create variants of CVS the best image quality was the true requirement. ${ }^{13,14}$ The optimal interval was always confirmed using multi-planar reformatting images. On selected intervals, the main coronary veins were searched and analyzed.

All data were evaluated by 2 investigators experienced in MSCT of CVS, as well as in cardiac resynchronization system implantation. ${ }^{13,14}$

\section{Coronary Veins Nomenclature}

Based on previous data ${ }^{15,16}$ with some modifications the following nomenclature was used:

Coronary Sinus (CS) Originates from the great cardiac vein and drain in the atrio-ventricular groove to the right atrium by CS ostium. CS ostium is located in the posteroseptal right atrium. Sometimes CS ostium is covered by the Thebesian valve. ${ }^{9}$

Great Cardiac Vein (GCV) Originates in the anterior vein

\begin{tabular}{|lc|}
\hline $\begin{array}{l}\text { Table 1. Hemodynamic Characteristics of the Included } \\
\text { Patients }\end{array}$ & Hemodynamic parameters \\
& $63.42 \pm 7.98$ \\
Ejection fraction $(\%)$ & $143.64 \pm 42.98$ \\
EDV $(\mathrm{ml})$ & $56.10 \pm 35.92$ \\
ESD $(\mathrm{ml})$ & $87.29 \pm 19.51$ \\
Stroke volume $(\mathrm{ml})$ & $5.39 \pm 1.56$ \\
Cardiac output $(\mathrm{L} / \mathrm{min})$ & $137.19 \pm 44.55$ \\
Myocardial mass $(\mathrm{g})$ & $129.94 \pm 42.35$ \\
Myocardial volume $(\mathrm{ml})$ & \\
\hline
\end{tabular}

(AV) near the left anterior descending artery and end as CS in the ostium vein of Marshall.

Middle Cardiac Vein (MCV) Originates close to the apex of the heart and follows the posterior interventricular groove toward the base.

Posterior Vein (PV) (Referred Also as Left Marginal Vein) Originates either from the posterior or lateral aspects of the left ventricle and drain into the $C S$.

Posterolateral Vein (PLV) Fifteen degree area of lateral ventricle between $\mathrm{PV}$ and lateral vein (LatV). Usually drains into the CS. ${ }^{13}$

Lateral Vein (LatV) It runs in the lateral wall of the left ventricle to terminate in the CS or great cardiac vein.

Anterolateral Vein (ALV) Fifteen degree area of lateral ventricle between $\mathrm{AV}$ and LatV. Usually drains into the great cardiac vein. ${ }^{13}$

Anterior Vein (AV) (Referred Also as Anterior Interventricular Vein) Originates at the lower or middle third of the anterior interventricular groove. It follows the groove toward the base of the heart and then turns posterior at the atrioventricular groove to enter the great cardiac vein.

Vein of Marshall (VM) (Referred Also as Oblique Vein of the Left Atrium) It is a small vessel, which descends obliquely on the back of the left atrium and ends in the CS near its left extremity.

To facilitate nomenclature with MSCT, the analyzing matrix was created (Figure 1). This matrix was used on flat images, which were created from 5 to 6 3D images by using a specific graphic software. Each matrix consists of 15 areas. PV, LatV and ALV were defined as CRT target veins.

\section{Results}

The cardiac function parameters of the group examined are presented in Table 1. CS and middle cardiac vein were well visualized in all cases. AV was the most common vessel and

\begin{tabular}{|cccccccc|}
\hline \multicolumn{6}{|c|}{ Table 2. Common Variants of Coronary Venous System Which Were Found in This Research } \\
Variant & PV & PLV & LatV & ALV & AV & Number of cases & Frequency (\%) \\
1 & No & Yes & Yes & No & Yes & 26 & 13.1 \\
2 & Yes & Yes & Yes & No & Yes & 23 & 11.6 \\
3 & Yes & Yes & Yes & Yes & Yes & 18 & 9.1 \\
4 & No & Yes & Yes & Yes & Yes & 16 & 8.1 \\
5 & Yes & No & Yes & No & Yes & 16 & 8.1 \\
6 & No & No & Yes & No & Yes & 16 & 8.1 \\
7 & No & Yes & No & No & Yes & 13 & 6.6 \\
8 & Yes & No & Yes & Yes & Yes & 10 & 5.1 \\
9 & No & No & Yes & No & No & 10 & 5.1 \\
\hline
\end{tabular}

PV, posterior vein; PLV, posterolateral vein; LatV, lateral vein; ALV, anterolateral vein; $A V$, anterior vein; Yes, vein is present; No, vein is absent. 

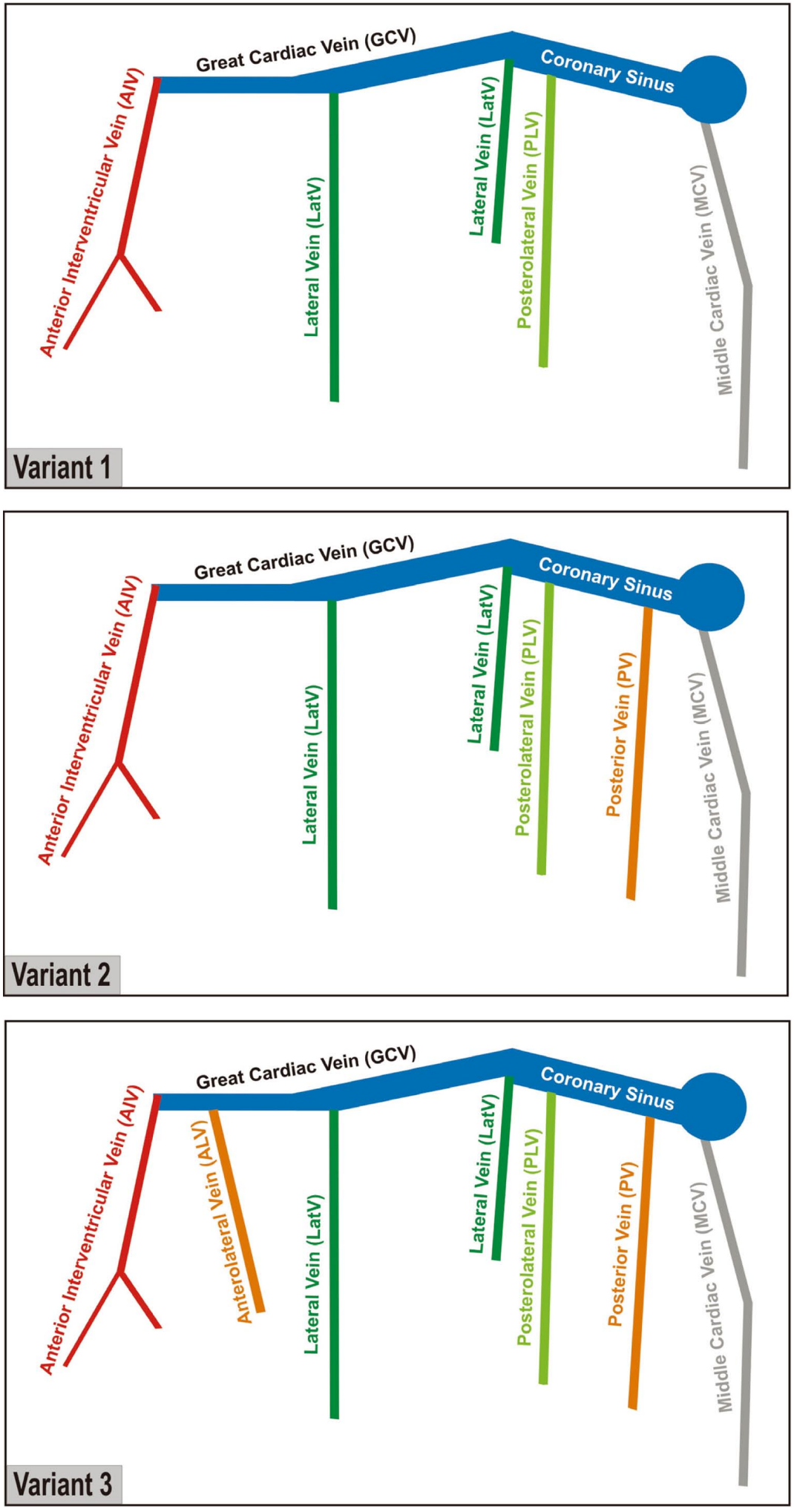

Figure 2. Schemes of 3 of the most common variants of coronary veins in presented research.

was visualized in $160 / 199$ cases $(80.4 \%)$. With the same frequency, the LatV was visualized, which is important as one of the target veins for cardiac resynchronization. Another important vein-PV-was identified in 124 patients $(62.3 \%)$. Alternatively PV was visible in 94 cases (47.2\%). The most seldom visible target veins was the ALV-69/199 (34.7\%).

Twenty-seven anatomical variants of CVS were identified, 9 of them are the most common. They were identified in 148 from 199 cases $(74.4 \%)$. Exact frequency of identified variants are presented in Table 2 . Schemes of 3 of the 
most common variants are presented (Figure 2). In 4 out of these 9 variants, a single coronary vein in the target area for CRT appeared, 2 target veins in 3 variants and 3 veins in 2 variants occurred. In 6 cases ( 2 seldom variants $-3.0 \%$ ) no veins in the target area were identified.

There was no correlation between occurrence of specific variants and cardiac function parameters.

\section{Discussion}

Although CVS seems to have a relatively low interest from a clinical point of view (if compared to the coronary arteries), during the past decade there are some groups of electrophysiologists for which this knowledge could be especially important. According to Vogt et al, 3 important aspects regarding CVS are most relevant. ${ }^{17}$ Access to the specific anatomical variants of the CS and handling of complex target vein anatomy are directly connected with CVS anatomy. Evaluation of CVS anatomy might be of special interest before the procedure CRT implantation. There is agreement that MSCT can precisely evaluate the anatomy of the heart. In the appropriateness criteria, the MSCT for non-invasive coronary vein mapping prior to the placement of a biventricular pacemaker is marked as $\mathrm{A}(8)$, so it is recommended. ${ }^{18}$

Coronary venous anatomy is highly variable. ${ }^{19-21}$ The first author who described coronary venous variations in detail by means of invasive angiography was Tori in 1952.22 Since then, mostly anatomo-pathological papers occurred. Some papers which described anatomical variant of coronary veins by using other methods were published. Gilard at al examined 100 patients with venous phase in analysis during coronary angiography. The results indicated that MCV and GCV (or CS) were consistently present on all venograms. ${ }^{21}$ They pointed out an interindividual variation of coronary veins with a special emphasis placed on PV. They concluded that in less then $5 \%$ of patients, the absence of target veins (in their study posterior and lateral) might practically limit the ability of left ventricle lead implantation. Despite different techniques of visualization, the results are similar to that presented in of our investigations - we also confirmed huge variability of CVS with the highest variability of PV. We also confirmed that in a small proportion of patients no veins in the target area can be found (6 patients; $3 \%$ of all).

Abbara et al were the first investigators who used MSCT for CVS evaluations by using a 16 slice scanner. ${ }^{23}$ They noticed that in $7.4 \%$ of cases no LatV could be found and in $20.4 \%$ of cases no PV could be found; however, in all included patients the posterior or LatV was visualized. In our study, the LatV was absent in almost $20 \%$ and PV in $53 \%$ of cases. The discrepancy between findings might result from differences in the nomenclature, as we recognized additional $\mathrm{PV}$, which might correspond to the PV in the study of Abbara et al.

Another study using a 16 slice scanner was performed by Jongbloed et al. ${ }^{24}$ As most authors found, CS was well visualized in all cases. PV was visualized in $86-90 \%$. They designated all included patients to 1 of 3 anatomical groups. They used modified variants proposed previously by von Lüdinghausen. ${ }^{19}$ As this division was strictly anatomical, in the presented work a special emphasis was placed on finding variants based on characteristic features for electrophysiological (as CRT implantation) purposes. It is difficult to compare those 2 different kinds of classification.

A Japanese study performed by Tada et al using 8 detectors CT scanner also showed the usefulness of MSCT in visualization of CVS. ${ }^{25}$ According to their results, the CS and middle cardiac vein were visible in all patients. The left marginal and PVs were visualized in $84 \%$ and $94 \%$, respectively. Japanese authors did not performed analysis of variants of coronary veins.

As presented investigations performed on 8 and 16 slice scanners, the use of 64 slice scanner (our research) might be more accurate due to higher resolution. Importance seemed to be placed also on the different methods of image post-processing to improve image quality. Novel techniques can play an important role in CVS visualization. ${ }^{26}$

Visualization of the CVS by using 64 slice scanners is sometimes difficult because the scan period is relatively long. As a consequence, coronary veins can potentially be opacified by the contrast agent. In the new era of ultra fast 256 or 320 detector-row scanners that require only one gantry rotation for coronary artery imaging, visualization of coronary veins can be better.

Huge anatomical variability of coronary veins was also confirmed by Bales. ${ }^{27}$ The author presented great cardiac vein variation based on intertwined variation found by a medical student during medical anatomy in one case.

In the era of percutaneus interventions, such as left ventricle lead implantation during cardiac resynchronization, the variability in the coronary venous anatomy is the real challenge. ${ }^{15}$ The correct interpretation of the coronary venous anatomy derived from such non-invasive methods, such as MSCT and magnetic resonance of the heart, can potentially facilitate cardiac resynchronization implantation, by understanding variations and clinical meaning.

\section{Study Limitations}

There were no patients with advanced heart failure included into this study. Most of patients were examined because of a suspicion of CAD and had normal or near normal ejection fraction.

Other limitations are related to MSCT as a method itself. Excluding patients with arrhythmias is a limitation, especially because they are very common among patients with heart failure. The dose of radiation is substantial, particularly that ECG-dependent dose modulation algorithm was not used. The amount of contrast agent used in this study is significant-some protocols with a lower rate than $5 \mathrm{ml} / \mathrm{s}$ were tested by us, but the results in coronary arteries visualization was unsatisfying - coronary veins visualization was added to routine coronary arteries visualization due to CAD suspicion. Patients might require a $\beta$-blocker use for HR reduction, which could be poorly tolerated and require continuous $\mathrm{HR}$ and blood pressure monitoring.

This is a descriptive anatomical study that can be useful for electrophysiologists. Success of CS cannulation is more based on the operator's skills and experience than on the findings in MSCT; however, pre-procedural visualization of CVS can potentially change procedure and/or treatment strategy.

\section{Conclusions}

For invasive cardiologists, knowledge about CVS anatomy could add value before and during electrophysiology procedures. Most of the examined patients have at least one vein in the target area for CRT. Huge anatomical variability of CVS strengthen the role of MSCT in visualization CVS before CRT implantation. In patients with no coronary vein in the target area, renouncement from intravenous implantation of CRT device should be considered. 


\section{Acknowledgments}

The authors are indebted to the radiology technologists for their technical support.

\section{References}

1. Fujii B, Takami M. Normalization of left ventricular function following cardiac resynchronization therapy: Left bundle branch block as a potential etiology of dilated cardiomyopathy. Circ J 2008; 72: $1030-1033$.

2. Cleland JG, Calvert MJ, Verboven Y, Freemantle N. Effects of cardiac resynchronization therapy on long-term quality of life: An analysis from the CArdiac Resynchronization-Heart Failure (CAREHF) study. Am Heart J 2009; 157: 457-466.

3. Rossi A, Rossi G, Piacenti M, Startari U, Panchetti L, Morales MA. The current role of cardiac resynchronization therapy in reducing mortality and hospitalization in heart failure patients: A meta-analysis from clinical trials. Heart Vessels 2008; 23: 217-223.

4. Moss AJ. What we have learned from the family of multicenter automatic defibrillator implantation trials. Circ J 2010; 74: 1038 1041.

5. Tanaka Y, Tada H, Yamashita E, Sato C, Irie T, Hori Y, et al. Change in blood pressure just after initiation of cardiac resynchronization therapy predicts long-term clinical outcome in patients with advanced heart failure. Circ J 2009; 73: 288-294.

6. Worley SJ. How to use balloons as anchors to facilitate cannulation of the coronary sinus left ventricular lead placement and to regain lost coronary sinus or target vein access. Heart Rhythm 2009; 6: $1242-1246$.

7. Luedorff G, Grove R, Kranig W, Thale J. Different venous angioplasty manoeuvres for successful implantation of CRT devices. Clin Res Cardiol 2009; 98: 159-164.

8. Osman F, Kundu S, Tuan J, Pathmanathan RK. Use of coronary venous angioplasty to facilitate optimal placement of left ventricular lead during CRT. Pacing Clin Electrophysiol 2009; 32: 281-282.

9. Mak GS, Hill AJ, Moisiuc F, Krishnan SC. Variations in Thebesian valve anatomy and coronary sinus ostium: Implications for invasive electrophysiology procedures. Europace 2009; 11: 1136-1137.

10. Silver MA, Rowley NE. The functional anatomy of the human coronary sinus. Am Heart J 1988; 115: 1080-1084.

11. Van de Veire NR, Schuijf JD, De Sutter J, Devos D, Bleeker GB, de Roos A, et al. Non-invasive visualization of the cardiac venous system in coronary artery disease patients using 64-slice computed tomography. J Am Coll Cardiol 2006; 48: 1832-1838.

12. Lumia $\mathrm{D}$, Laganà $\mathrm{D}$, Canì $\mathrm{A}$, Mangini $\mathrm{M}$, Giorgianni $\mathrm{A}$, Cafaro $\mathrm{T}$, et al. MDCT evaluation of the cardiac venous system. Radiol Med 2009; 114: 837-851.

13. Mlynarski R, Sosnowski M, Wlodyka A, Kargul W, Tendera M. A user-friendly method of cardiac venous system visualization in 64slice computed tomography. Pacing Clin Electrophysiol 2009; 32: $323-329$.

14. Mlynarski R, Sosnowski M, Wlodyka A, Chromik K, Kargul W, Tendera M. Optimal image reconstruction intervals for noninvasive visualization of the cardiac venous system with a 64-slice computed tomography. Int J Cardiovasc Imaging 2009; 25: 635-641.

15. Christiaens L, Ardilouze P, Ragot S, Mergy J, Allal J. Prospective evaluation of the anatomy of the coronary venous system using multidetector row computed tomography. Int J Cardiol 2008; 126: 204-208.

16. Meisel E, Pfeiffer D, Engelmann L, Tebbenjohanns J, Schubert B, Hahn S, et al. Investigation of coronary venous anatomy by retrograde venography in patients with malignant ventricular tachycardia. Circulation 2001; 104: 442-447.

17. Vogt J, Heintze J, Hansky B, Güldner H, Buschler H, Horstkotte D. Implantation: Tips and tricks-the cardiologist's view. Eur Heart $J$ Suppl 2004; 6: D47-D52.

18. Hendel RC, Patel MR, Kramer CM, Poon M, Hendel RC, Carr JC, et al. ACCF/ACR/SCCT/SCMR/ASNC/NASCI/SCAI/SIR 2006 appropriateness criteria for cardiac computed tomography and cardiac magnetic resonance imaging: A report of the American College of Cardiology Foundation Quality Strategic Directions Committee Appropriateness Criteria Working Group, American College of Radiology, Society of Cardiovascular Computed Tomography, Society for Cardiovascular Magnetic Resonance, American Society of Nuclear Cardiology, North American Society for Cardiac Imaging, Society for Cardiovascular Angiography and Interventions, and Society of Interventional Radiology. J Am Coll Cardiol 2006; 48: $1475-1497$.

19. von Lüdinghausen $M$. The venous drainage of the human myocardium. Adv Anat Embryol Cell Biol 2003; 168: 1-104.

20. Ortale JR, Marquez CQ. Anatomy of the intramural venous sinuses of the right atrium and their tributaries. Surg Radiol Anat 1998; 20: $23-29$.

21. Gilard M, Mansourati J, Etienne Y, Larlet JM, Truong B, Boschat $\mathrm{J}$, et al. Angiographic anatomy of the coronary sinus and its tributaries. Pacing Clin Electrophysiol 1998; 21: 2280-2284.

22. Tori G. Radiological visualization of the coronary sinus and coronary veins. Acta Radiol 1951; 36: 405-410.

23. Abbara S, Cury RC, Nieman K, Reddy V, Moselewski F, Schmidt $\mathrm{S}$, et al. Noninvasive evaluation of cardiac veins with 16-MDCT angiography. Am J Roentgenol 2005; 185: $1001-1006$.

24. Jongbloed MR, Lamb HJ, Bax JJ, Schuijf JD, de Roos A, van der Wall EE, et al. Noninvasive visualization of the cardiac venous system using multislice computed tomography. J Am Coll Cardiol 2005; 45: 749-753.

25. Tada H, Kurosaki K, Naito S, Koyama K, Itoi K, Ito S, et al. Threedimensional visualization of the coronary venous system using multidetector row computed tomography. Circ J 2005; 69: 165170.

26. Yamada M, Jinzaki M, Kuribayashi S, Sato K, Tanami Y, Fukumoto $\mathrm{K}$, et al. Novel post-processing image for the visualization of the coronary sinus by multidetector-row computed tomography before cardiac resynchronization therapy: Edge-enhanced image. Circ J 2008; 72: 487-488.

27. Bales GS. Great cardiac vein variations. Clin Anat 2004; 17: 436443. 